\title{
Fractional Stochastic Field Theory
}

\author{
Juha Honkonen ${ }^{1, \star}$ \\ ${ }^{1}$ Finnish National Defence University, Helsinki, Finland
}

\begin{abstract}
Models describing evolution of physical, chemical, biological, social and financial processes are often formulated as differential equations with the understanding that they are large-scale equations for averages of quantities describing intrinsically random processes. Explicit account of randomness may lead to significant changes in the asymptotic behaviour (anomalous scaling) in such models especially in low spatial dimensions, which in many cases may be captured with the use of the renormalization group. Anomalous scaling and memory effects may also be introduced with the use of fractional derivatives and fractional noise. Construction of renormalized stochastic field theory with fractional derivatives and fractional noise in the underlying stochastic differential equations and master equations and the interplay between fluctuation-induced and built-in anomalous scaling behaviour is reviewed and discussed.
\end{abstract}

\section{Introduction}

Description of evolution at the kinetic stage is usually expressed in the form of differential equations with the understanding that they are large-scale equations for averages of quantities describing intrinsically random processes. Account of randomness may lead to significant changes in the asymptotic behaviour in such models especially in low spatial dimensions. An important example of changes in the large-scale behaviour is anomalous diffusion, which is deviation of the mean-square path-length of a random walker from the usual linear growth in time. Anomalous diffusion may occur, for instance, due to random advection, random walks with power-law falloff of the step-length distribution or long-tailed distribution of waiting times between consecutive steps. In case of interactions between fluctuations of densities anomalous diffusion may occur due to large-scale fluctuations even in the case of short-range interactions and correlations in the underlying kinetic model as in models of critical dynamics and stochastic transport models. This report is concentrated on the anomalous scaling behaviour in diffusion-limited reactions brought about by powerlike distribution of step length and waiting times.

Lévy flights are a generalization of random walks with a powerlike falloff of the step-length distribution $p(l) \propto l^{-1-\sigma}$ characterized by the step index $\sigma$ assuming values $0<\sigma<2$. In the generic case leading to anomalous diffusion due to spatial effects, the probability density function of the position $\mathbf{r}$ of a test particle obeys the Fokker-Planck equation

$$
\frac{\partial P}{\partial t}=-D^{\prime}\left(-\nabla^{2}\right)^{\sigma / 2} P+D \nabla^{2} P
$$

\footnotetext{
^e-mail: juha.honkonen@mil.fi
} 
Here, the fractional power of $\nabla^{2}$ may be defined through the Fourier transform i.e. by writing the diffusion operator in the Fourier space as

$$
-D^{\prime}\left(-\nabla^{2}\right)^{\sigma / 2} P+D \nabla^{2} P \rightarrow-D^{\prime}\left(\mathbf{k}^{2}\right)^{\sigma / 2} P-D \mathbf{k}^{2} P .
$$

In the position space the fractional power of the Laplace operator $\nabla^{2}$ may be defined with the use of an integral operator (Riesz derivative). In this case the fractional differential operator must be understood in terms of distribution theory [1]. The ordinary diffusion term $D \nabla^{2} P$ in (1) is brought about by the small-scale part of the step distribution under renormalization and must be taken into account, when the step index $\sigma \rightarrow 2$. For fixed $\sigma<2$ the ordinary diffusion term is irrelevant by power counting [2].

In epidemic models there are situations in which an infected individual can infect other individuals only after a certain incubation time (waiting time) [3] giving rise to long tails in waiting-time distribution of the form

$$
p(\Delta t) \propto(\Delta t)^{-1-\alpha} .
$$

Long tails in waiting times give rise to memory in system dynamics. Memory effects may be described by integral operators, which in case of powerlike asymptotic behaviour of waiting-time distribution gives rise to fractional differentiation and integration.

\section{Fractional derivatives}

There are different definitions of fractional derivatives on a time interval. Popular choices are the fractional derivative of Riemann-Liouville

$$
\left(\mathcal{D}_{+}^{\alpha} f\right)(t)=\frac{1}{\Gamma(1-\alpha)} \frac{d}{d t} \int_{-\infty}^{t} \frac{f\left(t^{\prime}\right) d t^{\prime}}{\left(t-t^{\prime}\right)^{\alpha}}
$$

and the fractional derivative of Marchaud

$$
\left(\mathbf{D}_{+}^{\alpha} f\right)(t)=\frac{\alpha}{\Gamma(1-\alpha)} \int_{-\infty}^{t} \frac{f(t)-f\left(t^{\prime}\right)}{\left(t-t^{\prime}\right)^{1+\alpha}} d t^{\prime},
$$

whose definitions are quoted here for the whole time axis [1]. Fractional derivatives (2) and (3) are not equivalent, in general, but they coincide on functions "decent enough" [1]. From the point of view of construction of perturbation expansion, the basic property of the fractional derivative is its Fourier transformation:

$$
\mathcal{F}\left(\mathcal{D}_{+}^{\alpha} f\right)(\omega)=(-i \omega)^{\alpha} \mathcal{F}(f)(\omega), \quad \operatorname{Re} \alpha \geq 0,
$$

where

$$
\mathcal{F}(f)(\omega)=\int_{-\infty}^{\infty} e^{i \omega t} f(t) d t .
$$

Relation (4) allows to use standard Feynman rules in the Martin-Siggia-Rose approach to stochastic problems [4].

There are different ways to introduce the temporal fractional derivative into a kinetic equation. The most straightforward way is to replace the usual time derivative by a fractional derivative, i.e. instead of (1) write $(0<\alpha \leq 1)$

$$
\left(\mathcal{D}_{+}^{\alpha} P\right)(t)=-D^{\prime}\left(-\nabla^{2}\right)^{\sigma / 2} P+D \nabla^{2} P .
$$


In this case, however, the quantity $P$ cannot be interpreted as a (probability) density [5], as in (1).

Conservation of probability imposes conditions on the structure of the Fokker-Planck equation and the consistent way to proceed appears to be to apply the fractional time derivative to the righthand-side operator of the Fokker-Planck equation instead of replacing the usual time derivative on the left-hand side by a fractional time derivative [6]. In the case of Lévy flights this corresponds to representation

$$
\frac{\partial P}{\partial t}=\left(\mathcal{D}_{+}^{1-\alpha}\left\{-D^{\prime}\left(-\nabla^{2}\right)^{\sigma / 2} P+D \nabla^{2}\right\}\right)(t) .
$$

instead of the simple-minded (5).

The Green function of the fractional differential equation

$$
\left(\mathcal{D}_{+}^{\alpha} \Delta_{1}\right)(t)+D^{\prime}\left(\nabla^{2}\right)^{\sigma / 2} \Delta_{1}=0
$$

given by the derivative of the Mittag-Leffler function $E_{\alpha}(z)$ (see, e.g. [7]) as

$$
\Delta_{1}(t, \mathbf{k})=\int_{s_{0}-i \infty}^{s_{0}+i \infty} \frac{e^{s t} d s}{s^{\alpha}+D^{\prime} k^{\sigma}}=\theta(t) \alpha t^{\alpha-1} E_{\alpha}^{\prime}\left(-D^{\prime} k^{\sigma} t^{\alpha}\right)
$$

is singular in the limit $t \rightarrow 0$ so that a proper initial condition cannot be set and the normalization of the density is not conserved. On the contrary, the propagator $\Delta_{2}$ of the problem (6) is the Green function of the fractional differential equation

$$
\frac{\partial \Delta_{2}}{\partial t}+D^{\prime}\left(\nabla^{2}\right)^{\sigma / 2}\left(\mathcal{D}_{+}^{1-\alpha} \Delta_{2}\right)(t)=0
$$

given by the the Mittag-Leffler function as

$$
\Delta_{2}(t, \mathbf{k})=\int_{s_{0}-i \infty}^{s_{0}+i \infty} \frac{e^{s t} s^{\alpha-1} d s}{s^{\alpha}+D^{\prime} k^{\sigma}}=\theta(t) E_{\alpha}\left(-D^{\prime} k^{\sigma} t^{\alpha}\right)
$$

has a finite limit at $t \rightarrow 0: \Delta_{2}(t, \mathbf{k}) \rightarrow 1$ and also obeys the normalization condition $\Delta_{2}(t, 0)=1$. Therefore, the physically correct setup of the problem is given by (6).

\section{Kinetic equation}

It is instructive to start the construction of the field theory of fluctuations from the (deterministic) nonlinear kinetic equation for the expected value of the fluctuating quantity. The consistent form of the kinetic equation with fractional time derivatives is of the form

$$
\frac{\partial \varphi}{\partial t}=\mathcal{D}_{+}^{1-\alpha} V(\varphi)=-K \mathcal{D}_{+}^{1-\alpha} \varphi+\mathcal{D}_{+}^{1-\alpha} U(\varphi)+\mathcal{D}_{+}^{1-\alpha} \tilde{A},
$$

where $\tilde{A}$ is - for the time being - a fixed external field.

The nonlinear differential equation (9) allows for the tree-graph solution explicitly depending on initial conditions and coefficient functions and implicitly on boundary conditions through the Green function of the linear problem. All these conditions may contain randomness, whose effect on the solution is conveniently calculated with the use of the compact functional form of the tree-graph solution. 
Let $\varphi[\tilde{A}]$ be solution of the generic fractional kinetic equation (9). Define generating function of solutions of the kinetic equation

$$
G(A)=e^{A \varphi[\tilde{A}]} .
$$

The linear part of the right-hand side of (9) yields the Mittag-Leffler propagator

$$
\Delta_{12}^{M L}(t, \mathbf{k})=\theta(t) E_{\alpha}\left[-\left(D k^{2}+D_{\sigma} k^{\sigma}\right) t^{\alpha}\right] .
$$

Perturbation expansion for the generating functional is conveniently expressed in the functionaldifferential form as the S-matrix functional [8]

$$
G(A)=\left.\exp \left(\frac{\delta}{\delta \varphi} \Delta_{12}^{M L^{\prime}} \frac{\delta}{\delta \tilde{\varphi}}\right) \exp \left[\tilde{\varphi} \mathcal{D}_{+}^{1-\alpha} U(\varphi)+\tilde{\varphi} \mathcal{D}_{+}^{1-\alpha} \tilde{A}+A \varphi\right]\right|_{\tilde{\varphi}=\varphi=0}
$$

where $\Delta_{12}^{M L^{\prime}}\left(t, \mathbf{x} ; t, \mathbf{x}^{\prime}\right) \equiv 0$. It should be noted that this condition is only a shorthand for the normal form of the interaction functional, the latter meaning that closed loops of single propagators are absent in the graphical representation of perturbation expansion (see [9] for details). It should be emphasized that (12) is a functional representation of the solution of a deterministic nonlinear equation. In the next section it will be used to construct the solution of a stochastic differential equation by simply averaging over the probability distribution of the external field. The iterative solution of the equation is unambiguous. The ostensible ambiguity which arises, when representation (12) is derived, is the same both in the deterministic and in the stochastic case [10] and thus is a property of the functional representation and not a consequence of the stochastic nature of the problem.

Renormalization is almost inevitable step in the analysis of a field theory. Representation (12) has the drawback that the interaction functional is nonlocal in time, in which situation the standard procedures of the field-theoretic renormalization are inapplicable.

This situation may be cured with the use of integration by parts in the interaction term

$$
\tilde{\varphi} \mathcal{D}_{+}^{1-\alpha} U(\varphi)=U(\varphi) \mathcal{D}_{-}^{1-\alpha} \tilde{\varphi}=\int d t U(\varphi(t))\left(-\frac{1}{\Gamma(\alpha)} \frac{\mathrm{d}}{\mathrm{d} t} \int_{t}^{\infty} d u \frac{\tilde{\varphi}(u)}{(t-u)^{1-\alpha}}\right)
$$

In terms of the new variable $\tilde{\phi}=\mathcal{D}_{-}^{1-\alpha} \tilde{\varphi}$ the generating function

$$
G(A)=\left.\exp \left(\frac{\delta}{\delta \varphi} \Delta_{12}^{\alpha} \frac{\delta}{\delta \tilde{\phi}}\right) \exp [\tilde{\phi} U(\varphi)+\tilde{A} \tilde{\phi}+A \varphi]\right|_{\tilde{\phi}=\varphi=0}
$$

contains a local interaction functional and the usual rules of renormalization apply. The presence of fractional derivatives brings about some new features, however, which will be discussed a bit later.

\section{Langevin equation}

Fractional Langevin equation is the fractional kinetic equation

$$
\frac{\partial \varphi}{\partial t}=\mathcal{D}_{+}^{1-\alpha} V(\varphi)=-K \mathcal{D}_{+}^{1-\alpha} \varphi+\mathcal{D}_{+}^{1-\alpha} U(\varphi)+\mathcal{D}_{+}^{1-\alpha} f,
$$

with the white-in-time Gaussian noise

$$
\left\langle f(t, \mathbf{x}) f\left(t^{\prime}, \mathbf{x}^{\prime}\right)\right\rangle=\delta\left(t-t^{\prime}\right) D\left(\mathbf{x}-\mathbf{x}^{\prime}\right), \quad\langle f\rangle=0 .
$$


The generating function of solutions of this equation with fixed $f$ is (14) with the substitution $\tilde{A} \rightarrow f$. Integration of the generating functional over noise yields the functional-differential representation for the generating function of the Langevin problem (15), (16)

$$
G(A)=\left.\exp \left(\frac{\delta}{\delta \varphi} \Delta_{12}^{\alpha}{ }^{\prime} \frac{\delta}{\delta \tilde{\phi}}\right) \exp \left[\tilde{\phi} U(\varphi)+\frac{1}{2} \tilde{\phi} D \tilde{\phi}+A \varphi\right]\right|_{\tilde{\phi}=\varphi=0} .
$$

With the use of the identity

$$
\exp \left(\frac{\delta}{\delta \varphi} \Delta_{12}^{\alpha}{ }^{\prime} \frac{\delta}{\delta \tilde{\phi}}\right)=\int \mathcal{D} \phi \int \mathcal{D} \tilde{\varphi} \exp \left[\tilde{\varphi}\left(-\mathcal{D}_{+}^{\alpha}-K\right) \phi+\phi \frac{\delta}{\delta \varphi}+\tilde{\varphi} \frac{\delta}{\delta \tilde{\phi}}\right]
$$

representation (17) gives rise to the functional integral with the de Dominicis-Janssen dynamic action of quite standard form, let alone the fractional time derivative:

$$
G(A)=\int \mathcal{D} \phi \int \mathcal{D} \tilde{\varphi} \exp \left[\tilde{\varphi}\left(-\mathcal{D}_{+}^{\alpha}-K\right) \phi+\tilde{\varphi} U(\phi)+\frac{1}{2} \tilde{\varphi} D \tilde{\varphi}+A \phi\right] .
$$

\section{Renormalization}

As spatial fluctuations in the fractional diffusion equations give rise to the Laplace operator, the usual time derivative is often generated by fluctuations as well in field-theoretic models generated by Langevin equations. Thus, the generic propagator in the Langevin problem is [3]

$$
\Delta_{12}^{\alpha}(s, \mathbf{k})=\frac{1}{c_{1} s+c_{\alpha} s^{\alpha}+D k^{2}+D_{\sigma} k^{\sigma}} .
$$

Identification of canonical scaling dimensions and power counting in renormalization requires homogeneity of the propagator. Therefore, two terms in the denominator of the generic propagator (18) should be singled out to the free-field part of the dynamic action, which gives rise to the canonical dimensions of fields and the bare propagator of renormalized perturbation theory. The remaining two terms are then prescribed to the interaction and the canonical dimensions of their coefficient assume values corresponding to this choice. At first sight it would seem that the generic propagator (18) allows for several choices for the bare propagator. Analysis of similar situations in the field theory of static critical phenomena has revealed, however, that the consistent way to proceed is to separate the fractional terms to interaction $[11,12]$

$$
\begin{aligned}
& S_{0}=\tilde{\varphi}\left(-c_{1} \partial_{t}+D \nabla^{2}\right) \phi+\frac{1}{2} \tilde{\varphi} D \tilde{\varphi}, \\
& S_{I}=\tilde{\varphi}\left(-c_{\alpha} \mathcal{D}_{+}^{\alpha}-D_{\sigma}\left(-\nabla^{2}\right)^{\sigma / 2}\right) \phi+\tilde{\varphi} U(\phi) .
\end{aligned}
$$

The other way round produces renormalized theory with divergences in the limit $\sigma \rightarrow 2, \alpha \rightarrow 1$. It should be noted, however, that there are cases, when the usual differential operators are not generated by renormalization (e.g. the diffusion-limited coagulation model $A+A \rightarrow A$ ) and the propagator must be used in the Mittag-Leffler form (11) [13].

It is a fundamental rule of the theory of renormalization that nonlocal terms of the dynamic action are not renormalized at all (see, e.g., [2]). This property has been the source of a great deal of confusion in the stochastic field theory. A detailed analysis of this issue has been given on the example of renormalization of the stochastic Navier-Stokes equation in $[14,15]$. The crucial consequence in the 
$\mathrm{RG}$ analysis of the fractional Langevin problem is that the fractional diffusion constant and the coefficient of the fractional time derivative are not renormalized and therefore the corresponding terms of the dynamic actio are not changed due to fluctuations. However, the fractional terms contribute to the renormalization of the local terms of the action. This gives rise to the phenomenon of generation terms: contributions corresponding to the local terms of the dynamic action are produced by renormalization (i.e. large-scale fluctuations) even if they were absent in the original action. Relevance of these contributions cannot be determined a priori: renormalization brings about changes in scaling dimensions of local terms and the assessment of relevance of fractional terms is possible only, when the effect of fluctuations to scaling of local terms has been calculated.

Splitting of the generic propagator (18) to the bare propagator and interaction quadratic in fields according to (19), (20) generates Feynman graphs with effective propagators with the structure

$$
\frac{\left(c_{\alpha} s^{\alpha}+D_{\sigma} k^{\sigma}\right)^{n}}{\left(c_{1} s+D k^{2}\right)^{n+1}} .
$$

Renormalization is carried out in the logarithmic theory. To assess the interplay of local and fractional terms deviations of the order of fractional derivatives from the integer order of the ordinary derivatives are assumed small. In practical calculations it is almost always the case that deviation from the critical dimension $\varepsilon=d_{c}-d$ is used as a regulator of UV-divergences of the field theory. In this case small $1-\alpha, 2-\sigma$ are analytic regulators and analytic renormalization is called for in a consistent renormalization procedure.

In the usual models of critical dynamics there is a single regulator $\varepsilon$ and the scheme of minimal subtractions (MS) is effective and popular (see, e.g. [2]). In case of several regulators the ray scheme is widely used: in this scheme all regulators are assumed to be of the same order. Therefore, just one regulator remains independent and the MS scheme is carried with respect to this independent regulator. Although popular, this approach is dubious to say the least.

The point is that in genuine analytic renormalization all singularities in regulators are removed from the graphs of perturbation theory.

All singularities are removed in the analytic renormalization: therefore, to be sure that renormalization is carried out consistently, the starting point should be the analytically regularized model logarithmic with respect to dimensional regulator as well. This is sometimes called the principle of maximum divergences. As in all renormalization schemes, there is a lot of freedom in the choice of any concrete version of the analytic renormalization. In particular, if the model is analytically regularized, renormalization may be carried out with the use of a scheme based on the subtraction of necessary coefficients of the Taylor expansion at a given value of external momenta of a divergent graph (the standard Bogolyubov-Parasyuk $R$ operation).

It is a fundamental result of the renormalization theory that the Bogolyubov-Parasyuk $R$ operation produces Green functions analytic in regulators [16]. The MS ray scheme does not share this property. A divergent (sub)graph gives rise to a factor of the form ( $n, m, l$-integers) [16]

$$
\frac{1}{n \varepsilon+m(2-\sigma)+2 l(1-\alpha)}=\frac{1}{\varepsilon} \frac{1}{(n+m \zeta+2 l \xi)} .
$$

Contribution of a graph to renormalization constant is a product of these factors over all subgraphs multiplied by a function analytic in $\varepsilon, 2-\sigma$ and $1-\alpha$ at the origin.

In the ray scheme the ratios $\zeta$ and $\xi$ in (22) are finite and divergences are poles in $\varepsilon$. In the MS scheme only contributions singular in $\varepsilon$ are extracted to the renormalization constants. In such an approach the ratio of two different expressions (22) is a finite quantity. Therefore, in the MS scheme applied with respect to $\varepsilon$ not all factors $(n+m \zeta+2 l \xi)^{-1}$ are removed. The result is that the 
coefficient functions of the renormalization group are meromorphic functions in $\varepsilon, 2-\sigma$ and $1-\alpha$, not analytic as they should be! This fact does not show at one-loop approximation, in which most of the results have been calculated. However, explicit two-loop results in the stochastic Navier-Stokes problem have substantiated the picture described here [15]. Moreover, in this problem it may be seen from the explicit pole structure similar to (22) that calculations in the MS ray scheme in high orders inevitably lead to contributions diverging at the physical values of the regulators. From here it follows that the MS ray scheme is at least dubious, if not completely inconsistent beyond the one-loop approximation. On the other hand, pure analytic renormalization is not well suited for calculation of renormalization constans essential in the usual renormalization-group analysis. Moreover, subtraction procedures based on analytic properties of coefficient functions of Feynman graphs are different in dimensional and analytic renormalization [16]. Therefore, in the case of combined dimensional and analytic regularization, which is the most relevant case in critical dynamics, the most straightforward procedure appears to be the subtraction scheme based on Taylor expansion at some normalization point.

\section{Conclusion}

In conclusion, construction of a renormalized field theory for the solution of a stochastic differential equation (Langevin equation) has been reviewed and analyzed. It is rather obvious that the result is stochastic field theory with fractional derivatives in the dynamic actions. In the analysis of renormalization of the model the important statement is that renormalization produces local counterparts of fractional derivatives with nontrivial scaling dimensions and these generation terms play an important rôle in the renormalization and the subsequent asymptotic analysis of the model. Another important property is that the fractional differential operators are not renormalized. Relevance of fractional and the corresponding usual derivatives to the asymptotic behaviour may be assessed only upon a renormalization group analysis of the renormalized model, in which the anomalous dimensions of the local differential operators are calculated. In has been pointed out that the consistent construction of the renormalization is produced directly, when the fractional differential operators are prescribed to the interaction in the perturbation theory. A renormalization procedure, which takes into account the interplay of fractional and usual differential operators inevitably leads to multi-parameter analytic regularization, possibly encompassing dimensional regularization as well. It has been pointed out that the popular in such cases ray scheme with minimal subtractions is not consistent beyond the oneloop order. It is conjectured that in multiloop calculations dimensional-analytic regularization with normalization-point subtractions is the consistent and convenient method to use.

\section{References}

[1] С.Г. Самко, А.А. Килбас, О.И. Маричев, Интегралы и производные дробного порядка и некоторые их приложения (Наука и техника, Минск, 1987); S.G. Samko, A.A. Kilbas, O.I. Marichev, Fractional Integrals and Derivatives. Theory and Applications (Gordon and Breach, Amsterdam, 1993)

[2] A.N. Vasil'ev, The Field Theoretic Renormalization Group in Critical Behavior Theory and Stochastic Dynamics (Chapman \& Hall/CRC, Boca Raton, 2004)

[3] J. Adamek, M. Keller, A. Senftleben, H. Hinrichsen, J. Stat. Mech. P09002 (2005).

[4] P.C. Martin, E.D. Siggia, H.A. Rose, Phys. Rev. A 8, 423 (1973); C. De Dominicis, J. Phys. (Paris) 37, Suppl C1, 247 (1976); H.K. Janssen, Z. Phys. B 23, 377 (1976)

[5] H. Hinrichsen, J. Stat. Mech. P07006 (2007) 
[6] R. Metzler, E. Barkai, J. Klafter, Phys. Rev. Lett. 82, 3563 (1999).

[7] A. Erdélyi (ed.), Higher transcendental functions, volume III (McGraw-Hill, New York, 1981)

[8] M. Hnatič, J. Honkonen, T. Lučivjanský, Acta Physica Slovaca 66, 69 (2016)

[9] A.N. Vasiliev, Functional Methods in Quantum Field Theory and Statistical Physics (Gordon and Breach, Amsterdam, 1998)

[10] J. Honkonen, Theor. Math. Phys. 169, 1460 (2011)

[11] J. Honkonen, M.Yu. Nalimov, J. Phys. A: Math. Gen. 22, 751 (1989)

[12] J. Honkonen, J. Phys. A: Math. Gen. 23, 825 (1990)

[13] J. Honkonen, Fractional derivatives in stochastic reaction-diffusion problems. The 15th Small Triangle Meeting on theoretical physics (Institute of Experimental Physics SAS, Košice, 2014) 74-79

[14] L.Ts. Adzhemyan, J. Honkonen, M.V. Kompaniets, A.N. Vasil'ev, Phys. Rev. E 68, 055302(R) (2003)

[15] L.Ts. Adzhemyan, J. Honkonen, M.V. Kompaniets, A.N. Vasil'ev, Phys. Rev. E 71, 036305 (2005)

[16] О.И. Завьялов, Перенормированные диаграммы Фейнмана (Наука, Москва, 1979); O.I. Zavialov, Renormalized Quantum Field Theory (Kluwer, Dordrecht, 1990)] 